\title{
Fully divine and fully human: A bi-modal outline towards consistency
}

\author{
Jc Beall (University of Notre Dame) \\ A.J. Cotnoir (University of St Andrews)
}

June 4, 2020

\section{From Chalcedon to contradiction}

The Council of Chalcedon 451 affirms: Christ is fully divine and fully human, not mostly human and in-part divine, not mostly divine and in-part human, and not some other hybrid combination of divinity and humanity. Given classical conceptions of divinity and humanity, Chalcedon's account leads to apparent contradiction. This is what Richard Cross has dubbed 'the fundamental problem [of the incarnation]' [10, p. 453]. Example:

1. Christ is mutable. [Rationale: entailed by human nature.]

2. Christ is immutable. [Rationale: entailed by divine nature.]

3. Christ is mutable and immutable. [Rationale: 1-2, logic.]

Likewise, mutatis mutandis, for im-/passibility, im-/peccability, im-/materiality, and so on for the many pairs of properties either entailed by the two natures (viz., divine and human) or otherwise affirmed in christian creeds and orthodox theologies.

\section{$2 \quad$ Standard routes towards consistency}

Because the incarnation is at the very foundation of christian theology, the fundamental problem demands a response. One might accept that the apparent contradictions are veridical ${ }^{1}$ however, the dominant strategy, which we pursue here, seeks a logically consistent account of the incarnation, aiming to explain how the apparent contradictions of Christ are merely apparent. There are four, sometimes overlapping, routes towards consistency charted by Cross [10]. ${ }^{2}$

\footnotetext{
${ }^{1}$ Beall $[4,5]$ advances such a response, arguing that there are contradictions true of Christ.

${ }^{2}$ Other prominent discussions of the problem are many, including recently Marilyn McCord Adams' work [1], Oliver Crisp's work [8, 9], Thomas McCall's work [17], Sara Coakley's work [7] and references therein.
} 
- Reduplication: here, 'Christ is mutable' and/or 'Christ is immutable' are shorthand for QUA-adorned truths - namely, 'Christ-qua-human is mutable' or the like, where different strategies put the QUA device in different places. The error in the given derivation is that premises (1) and (2) are insufficiently expressed: the truths involve QUA adornments that undermine the steps to $(3) .^{3}$

- Relative identity: here, the identity relation peculiar to christology is a so-called relative one, famously explored by van Inwagen [20,21] and Martinich [16]. The key here is that while, per Chalcedon, Christ is the same person as the human Christ and the divine Christ, the latter two beings are not identical beings, and so can consistently have two contrary properties (i.e., properties the joint having of which entails a contradiction) as consistently as any two non-identical objects can each have exactly one of two contrary properties. The error in the given derivation rests on mistaken principles of theological identity relations. ${ }^{4}$

- Composition: here, the target predicates are (in effect) primarily applied to proper 'parts' (or aspects or whathaveyou) - in the most general sense of 'parts' - of Christ, and only derivatively to Christ. Example: Christ's physical body is mutable but no divine 'part' of Christ is mutable. Here, the contradiction fails because an object counts as mutable in virtue of one of its mutable parts but also counts as immutable in virtue of an immutable part - no more inconsistent than 'in-part green' and 'in-part red', applied to one object, are inconsistent. ${ }^{5}$

- Restriction: here, the idea is that either the classical theory of divinity, which entails (2) in the target derivation, or the classical theory of humanity, which entails (1), should be rejected, and in turn the derivation of target contradictions fails due to faulty initial premises. ${ }^{6}$

This list does not exhaust the theoretical landscape; ${ }^{7}$ there are other options. Our aim is to outline an option that takes seriously the modal features of the target properties (e.g., im-/mutable, im-/passable, etc.).

\footnotetext{
${ }^{3}$ Gorman [11] and Beall-Henderson [6] explore recent versions of this approach.

${ }^{4}$ Jedwab [14] explores recent versions of this approach.

${ }^{5}$ There is obviously a lot of room to have rival accounts along these lines, depending on notions of 'parts', composition (mereological or otherwise), metaphysics of natures, etc. Marmodoro and Hill [15] present a taxonomy. Pawl [18, 19] explores relevant issues at great length, developing a compositional but officially non-mereological account.

${ }^{6}$ The most recent account along these lines is in Michael Gorman's work [12].

${ }^{7}$ Another salient option is an epistemic-mystery direction: the apparent contradictions are inevitably apparent given our epistemic states; however, the truth, whatever it is, isn't because can't be - contradictory. Anderson [3] advances such an account.
} 


\section{$3 \quad$ Being explicit about possibility}

Many of the fundamental-problem properties involve implicit appeals to possibilities. ${ }^{8}$ This is the key to the target route towards consistency: the consistency of the incarnation is explained by the structure of possibilities induced by such a radical event. There are two core parts of the explanation: different modal operators, and the structure of modal space over which the given operators range.

For simplicity (but without loss of generality) we focus on the properties involved in the sample derivation: mutable and immutable. On their standard meanings, these properties are exemplified by an object $x$ just if it's possible that $x$ changes and, respectively, it's impossible that $x$ changes. On standard treatments of possibility, these amount to the conditions that there's some world at which $x$ changes and, respectively, there's no world at which $x$ changes. ${ }^{9}$ The current bi-modal option points to a distinction motivated by the target natures (viz., the divine nature and the human nature). Instead of recognizing only one modal operator at work in the christological affirmations, the idea is to recognize two, each tied to exactly one of the two different natures. A natural way to express the two operators explicitly invokes the given natures:

- It is divinely possible that...

- It is humanly possible that...

On the standard account of possibility operators, possibility is relative possibility in a two-fold sense: namely, that what is possible is in fact possible-at- $w$ for some world $w$, and that what's possible-at- $w$ is tied to a given 'access relation' which is a binary relation on some subset (possibly the entire set) of worlds in modal space.

The treatment of the two target possibility operators ties them to their own accessibility relations, say, $R^{d}$ for the divine-access relation and $R^{h}$ for the human-access relation. One relation picks out points of modal space that count as 'divinely possible' from a given world, and the other the points that are 'humanly possible' from a given world. (We say more about these relations in $\S 4$.)

Where $A$ is any sentence, and $\diamond_{d}$ and $\diamond_{h}$ the divine and human possibility operators, respectively, the standard truth conditions are in force: ${ }^{10}$

\footnotetext{
${ }^{8}$ It is an open question as to whether all problematic pairs are implicitly modal, and so whether the strategy pursued here is fully general. If some properties (e.g. im-/material) are fundamentally non-modal then additional tools are required in the final resolution. But by our lights, even if its application resolves only the most salient of the target contradictions, the utility of the proposed bi-modal strategy remains high. Hybrid approaches to theological problems are not at all uncommon, and putting another tool in the theological toolbox, at least by our lights, can only benefit theology more broadly.

${ }^{9}$ An important point: standard semantics for possibility operators do not require worlds but rather just some so-called point, where a point (be it a 'world', 'situation', 'time', 'evidential state', etc.) is something at which sentences can be true or false. ( $\$ 4$ carries this point forward.)

${ }^{10}$ No constraints are imposed on the given accessibility relations - including no requirement that they be reflexive, symmetric or transitive. More on this in $\S 4$.
} 
- $\diamond_{d} A$ is true at $w$ iff there's some $R^{d}$-accessible point $x$ at which $A$ is true.

- $\diamond_{d} A$ is false at $w$ iff for all $R^{d}$-accessible points $x, A$ is false at $x$.

- $\diamond_{h} A$ is true at $w$ iff there's some $R^{h}$-accessible point $x$ at which $A$ is true.

- $\diamond_{h} A$ is false at $w$ iff for all $R^{h}$-accessible points $x, A$ is false at $x$.

Moreover, one can use logical negation $\neg$ to define the corresponding notions of impossibility, namely,

- $\neg \diamond_{d} A$ is true at $w$ iff $\diamond_{d} A$ is false at $w$.

- $\neg \diamond_{h} A$ is true at $w$ iff $\diamond_{h} A$ is false at $w$.

The idea, then, is that Chalcedon demands true possibility claims, but - in light of the very different natures involved - does not demand that such claims be univocal. In particular, while
$5 . \diamond_{h}($ Christ changes $)$
6. $\neg \diamond_{h}($ Christ changes $)$

are clearly contradictory, and likewise while

7. $\diamond_{d}($ Christ changes $)$

8. $\neg \diamond_{d}$ (Christ changes $)$

are equally clearly contradictory, what's not clearly contradictory are the two fundamental but different possibility claims:

5. $\diamond_{h}($ Christ changes $)$

9. $\neg \diamond_{d}($ Christ changes $)$

And that's the promise of the bi-modal approach towards a consistent account.

\section{The structure of modal space}

So far we have said very little to explain the philosophical underpinnings of the two modal operators and their accessibility relations; the approach, as it stands, could use some additional metaphysical motivation. We want to stress that the details could be filled-in differently according to varying philosophical and theological sensibilities. The core modal solution requires only minimal modal constraints. ${ }^{11}$ Still, for concreteness and clarity, we put forward one (potential) metaphysical picture that makes sense of the approach.

\footnotetext{
${ }^{11}$ All that's required on the target account are three disjoint sets of points $D, H, W$, such that $D$ is the range of $R^{d}, H$ is the range of $R^{h}$, and the actual world is in $W$. This is illustrated below.
} 
Modality is concerned with possibilities. And on the usual story, modal operators looks at maximal possibilities - namely possible worlds. We begin with a set $W$ of possible worlds. Following [2, p. 224f], we treat (i.e., model) each world $w$ in $W$ as a maximal consistent set of propositions. The set is maximal in the sense that for every pair of mutually contradictory propositions, one member of that pair is in the set. The set is consistent in the sense that no mutually contradictory propositions are in the set. We use '@' as a name for the actual world, which is (modeled as) the set of all true propositions.

But possible worlds, so understood, do not exhaust the relevant possibilities; some (many) possibilities are not maximal in the given sense (above). On a standard usage of the term 'possibility', going back at least to Humberstone [13], a possibility can be any part of a possible world - including, but not limited to, the world itself. And this is the key to understanding our two modal operators. Each possible world in $W$ can be 'split', or partitioned, into two parts: the divine part and the created part. This is accomplished by dividing the set of propositions according to what they are about. If the proposition is about God as God is intrinsically, then it goes into the divine part of the world. If the proposition is about the created order, or about God in relation to that created order, then the proposition goes into the created part of the world. ${ }^{12}$ Each of these parts is a possibility - a consistent set of propositions (as any subset of a consistent set is consistent) - but not necessarily maximal. ${ }^{13}$ Let $D$ be the set of all divine possibilities, so understood, and $H$ be the set of all created possibilities, so understood.

In some worlds, the ones where God becomes incarnate, a divine person has both a divine and a created nature. ${ }^{14}$ This means there are divine and creaturely propositions involving one and the same person; the same subject appearing in both parts. Propositions like the one expressed by 'Christ is begotten' go into the divine part while the proposition expressed by 'Christ changes' goes into the creaturely part. But it is important to see that, in the case of divine incarnation, the same person may be the subject of both divine and creaturely propositions, and so may appear in possibilities in both $D$ and $H .{ }^{15}$

\footnotetext{
${ }^{12}$ While all creaturely propositions go into the created part, not all theological propositions go into the divine part. The division corresponds roughly to the traditional distinction between truths about God in se and truths about God ad extra.

${ }^{13}$ Though it could be: in worlds where God chooses not to create, the created part of a world is empty, and hence the divine part of such worlds is (modeled by) a maximally consistent set. The divine part of a world can never be empty, however, if God is a necessary being.

${ }^{14}$ There is no assumption here that incarnation worlds include only worlds with the incarnate Christ. If the traditional medieval doctrine that any person of the Trinity could have been incarnate is correct, then $W$ would include worlds with each of the three persons is incarnate. If, rather, the incarnation is ontologically constitutive of the Son (as on e.g. some readings of Barth), then the only incarnational worlds would have to include Christ. Thanks to an anonymous referee for discussion.

${ }^{15}$ The fact that a single entity is the subject of propositions at multiple possibilities does not entail that there's either $R^{d}$ access or $R^{h}$ access between those worlds. This is parallel to the case of quantified modal logics where transworld identity does not standardly entail accessibility for metaphysical modality. Thanks to an anonymous referee for raising the issue.
} 
Our access relations $R^{d}$ and $R^{h}$ can now be defined. From a given world $w$, the divine-access relation $R^{d}$ ranges only over the (set of all) divine possibilities: $R^{d}$ is a relation from $W$ to $D .{ }^{16}$ Similarly, the human-access relation $R^{h}$ ranges only over the (set of all) created possibilities: $R^{h}$ is a relation from $W$ to $H .{ }^{17}$ As with standard semantics for other modalities, the exact behavior (e.g., entailments) of the target operators $\diamond_{d}$ and $\diamond_{h}$ can depend on the properties (e.g. reflexivity, transitivity, etc.) one's theory imposes on the governing accessibility relations. For now, our basic outline imposes no constraints on the given relations - leaving exact details of such properties for theological debate.

\section{$5 \quad$ Towards a bi-modal solution}

Recall that all possibilities (all points in $D, H$, or $W$ ) are sets of propositions. An atomic proposition's being true-at-a-possibility is just that proposition's being in the set. Dually, an atomic proposition's being false-at-a-possibility is just that proposition's not being in the set. Extending beyond atomic propositions: the standard truth and falsity conditions for the logical operators apply; ${ }^{18}$ the key modal operators are defined via the truth and falsity clauses in $\S 3 .{ }^{19}$

The full shape of the solution is now available. The claim ' $\diamond_{h}$ (Christ changes)' is true at @ iff there's some $R^{h}$-accessible point (hence, some point $x$ in $H$ ) such that 'Christ changes' is true at $x$. There may be many creaturely possibilities at which 'Christ changes' is true, including the creaturely part of @. On the other hand, the claim ' $\neg \diamond_{d}$ (Christ changes)' is true at @ iff there's no $R^{d}$-accessible point (hence, no $y$ in $D$ ) such that 'Christ changes' is true at $y$. But since $y$ is in $D$ - that is, a divine possibility $-y$ is a set of propositions about God's intrinsic nature. Traditionally, change is never attributable to God in se, and so the claim 'Christ changes' will never be in the divine part of any world - and so ' $\neg \diamond_{d}$ (Christ changes)' is true at @. Hence, both immutability and mutability are made true (at the actual world), albeit relative to different modalities.

\footnotetext{
${ }^{16}$ In standard set-theoretic notation: $R^{d} \subseteq W \times D$. One might also want to add that every point in $D$ accesses every other point in $D$, or some other similar constraint on points in $D$.

${ }^{17} R^{h} \subseteq W \times H$. One might also want to add that every point in $H$ accesses every other point in $H$, or a similar constraint.

${ }^{18}$ For example:

- $A \wedge B$ is true-at- $x$ iff $A$ is true-at- $x$ and $B$ is true-at- $x$.

- $A \wedge B$ is false-at- $x$ iff $A$ is false-at- $x$ or $B$ is false-at- $x$.

- $\neg A$ is true-at- $x$ iff $A$ is false-at- $x$.

- $\neg A$ is false-at- $x$ iff $A$ is true-at- $x$.

All other truth-functional compounds can be defined as usual.

${ }^{19}$ Our aim in this paper is to present just the big-picture strategy, not to get into nitty-gritty logic, but we note that the intended definition of validity for the theory is truth preservation over worlds, not truth preservation over proper parts of worlds. Equivalently, validity is defined as absence of counterexample, where a counterexample to the argument from premises in $X$ to conclusion $A$ are 'whole possibilities' - that is, worlds - at which everything in $X$ is true but at which $B$ is untrue.
} 
On the bi-modal solution, the apparent contradiction of Christ is only apparent. We disambiguate the implicit modalities involved in the salient pairs of (modal) predicates, understanding one as ranging over sets of propositions involving God intrinsically and the other as ranging over sets of propositions involving God in relation to created natures - including Christ's own human nature. ${ }^{20}$ Mutability is truly attributed to Christ via the it is humanly possible that operator, which ranges over created possibilities; immutability is truly attributed to Christ via the it is divinely possible that operator, which ranges over divine possibilities.

\section{$6 \quad$ Summary}

The aim of this discussion has not been to advance a detailed solution to the fundamental problem of christology but rather to advance a strategy towards consistency, one that takes seriously the modal features inherent in (many versions of) the problem. The key lesson is twofold: first, the Chalcedonian demand for full divinity and full humanity is not a demand for univocal modalities. Two different modal operators, one ranging over divine possibilities and the other over creaturely possibilities, resolves the contradiction.

The bi-modal strategy towards consistency is novel and natural. Philosophers already recognize a number of modal operators: epistemic, deontic, temporal, logical, and nomological modalities. It's time for theologians to consider whether distinctively theological modalities do important theological work. ${ }^{21}$

\section{References}

[1] Marilyn McCord Adams. Christ and Horrors: The Coherence of Christology. Cambridge University Press, 2006.

[2] Robert Adams. Theories of actuality. Noûs, 8:211-231, 1979.

[3] James Anderson. Paradoxes in Christian Theology. Paternoster, Waynesboro, GA, 2007.

[4] Jc Beall. Christ - a contradiction. Journal of Analytic Theology, 7:400-433, 2019.

[5] Jc Beall. The Contradictory Christ. Oxford University Press, Oxford, 2020. In press.

\footnotetext{
${ }^{20}$ This aspect of the target approach might (might) reflect some of the desiderata and motivation - though not the details or implementation - of Michael Gorman's recent 'restrictivist' approach [12], work that focuses on various modal principles (without focusing on the modal space or semantics behind those principles).

${ }^{21}$ Acknowledgements: We are very grateful to an anonymous referee whose comments greatly improved this paper. Thanks also to ...[BLANK FOR BLIND REVIEW].
} 
[6] Jc Beall and Jared Henderson. A neglected qua solution to the fundamental problem of christology. Faith and Philosophy, 36, 2019.

[7] Sarah Coakley. What Chalcedon solved and didn't solve. In Stephen T. Davis, SJ Daniel Kendall, and SJ Gerald O'Collins, editors, The Incarnation, pages 143-163. Oxford University Press, Oxford, 2002.

[8] Oliver D. Crisp. Divinity and Humanity: The Incarnation Reconsidered. Cambridge University Press, Cambridge, UK, 2007.

[9] Oliver D. Crisp. God Incarnate: Explorations in Christology. T \& T Clark, New York, 2009.

[10] Richard Cross. The incarnation. In Thomas P. Flint and Michael Rea, editors, Oxford Handbook of Philosophical Theology. Oxford University Press, Oxford, 2011.

[11] Michael Gorman. Christological consistency and the reduplicative qua. Journal of Analytic Theology, 2:86-100, 2014.

[12] Michael Gorman. Classical theism, classical anthropology, and the christological coherence problem. Faith and Philosophy, 33(3):278-292, 2016.

[13] I. L. Humberstone. From worlds to possibilities. Journal of Philosophical Logic, 10(3):313-339, 1981.

[14] Joseph Jedwab. Against the Geachian theory of the trinity and incarnation. Faith and Philosophy, 32(2):125-145, 2015.

[15] Anna Marmodoro and Jonathan Hill. Composition models of the incarnation: Unity and unifying relations. Religious Studies, 46(4):469-488, 2010.

[16] A. P. Martinich. Logical operations. Journal of Religion, 58(2):169-181, 1978.

[17] Thomas McCall. An Invitation to Analytic Christian Theology. InterVarsity Academic Press, 2015.

[18] Timothy Pawl. In Defense of Conciliar Christology: A Philosophical Essay. Oxford University Press, Oxford, 2016.

[19] Timothy Pawl. In Defense of Extended Conciliar Christology: A Philosophical Essay. Oxford University Press, Oxford, 2019.

[20] Peter van Inwagen. And yet they are not three gods but one god. In Thomas V. Morris, editor, Philosophy and the Christian Faith, volume 5 of Notre Dame Studies in the Philosophy of Religion, pages 241-278. University of Notre Dame Press, 1988. 
[21] Peter van Inwagen. Not by confusion of substance, but by unity of person. In Richard Swinburne and Alan G. Padgett, editors, Reason and the Christian Religion: Essays in Honour of Richard Swinburne. Oxford University Press, 1994. 